\title{
Bronchiectasis due to an Ectopic Right Pulmonary Artery and Compression of the Superior Vena Cava between an Ascending Thoracic Aortic Aneurysm and Enlarged Right Pulmonary Artery
}

\author{
Hayri Ogul Mecit Kantarci \\ Department of Radiology, Medical Faculty, Ataturk University, Erzurum, Turkey
}

\section{Key Words}

Pulmonary arterial ectasia - Ascending aortic aneurysm .

Bronchiectasis - Computed tomography angiography

\begin{abstract}
Objective: The aim of this study was to report multidetector computed tomography (CT) imaging findings relating to segmental pulmonary arterial ectasia (PAE), which is a very rare vascular condition. Clinical Presentation and Intervention: A 70-year-old woman presented with shortness of breath and increased production of purulent sputum. Chest $\mathrm{CT}$ angiography revealed bronchiectasis secondary to compression of the anterior segment bronchus of the right upper lobe because of a segmental PAE. She also had compression of the superior vena cava between an ascending thoracic aortic aneurysm and an ectopic enlarged right pulmonary artery. Conventional right upper lobectomy and angioplasty were planned, but the patient refused surgical therapy. Conclusion: This report shows the importance of CT angiography in a case that involved an unusual coincidence of segmental PAE and ascending aortic aneurysm.
\end{abstract}

(c) 2016 S. Karger AG, Basel

\begin{tabular}{ll}
\hline KARGER & ( 2016 S. Karger AG, Basel \\
$\begin{array}{l}\text { E-Mail karger@karger.com } \\
\text { www.karger.com/mpp }\end{array}$ & $\begin{array}{l}\text { This is an Open Access article licensed under the terms of the } \\
\text { Creative Commons Attribution-NonCommercial 3.0 Un- } \\
\text { ported license (CC BY-NC) (www.karger.com/OA-license), } \\
\text { applicable to the online version of the article only. Distribu- } \\
\text { tion permitted for non-commercial purposes only. }\end{array}$
\end{tabular}

\section{Introduction}

Segmental pulmonary arterial ectasia (PAE) is a very rare vascular condition. It may be associated with pulmonary hypertension, congenital heart disease, connective tissue disease and vasculitis, and may cause tracheobronchial compression [1-3]. Compression of the superior vena cava between the ascending thoracic aortic aneurysm and segmental PAE is an unexpected pathology [3]. We hereby report a case of bronchiectasis resulting from a segmental PAE, and an unusual cause of superior vena cava compression.

\section{Case Report}

A 70-year-old woman presented with shortness of breath and an increased production of purulent sputum over the previous 6 weeks. Her past medical history included several episodes of respiratory tract infections during childhood. The patient had no history of smoking or congenital heart disease. She had experienced a gradual decrease in exercise tolerance for 7 years. She was admitted to our clinic for an acute exacerbation of chronic obstructive lung disease. On admission, right-sided infiltrates and volume loss in the upper lobe were noted. A physical examination revealed the patient to be clinically septic and in moderate respiratory distress. Her blood pressure was $130 / 80 \mathrm{~mm} \mathrm{Hg}$, her pulse rate was 105

Hayri Ogul, MD

Department of Radiology, Medical Faculty, Ataturk University Kazım Karabekir Mah. Terminal Cad., Site Polat Apt. B Blok, Kat 1, No. 2 TR-25240 Erzurum (Turkey)

E-Maildrhogul@gmail.com 

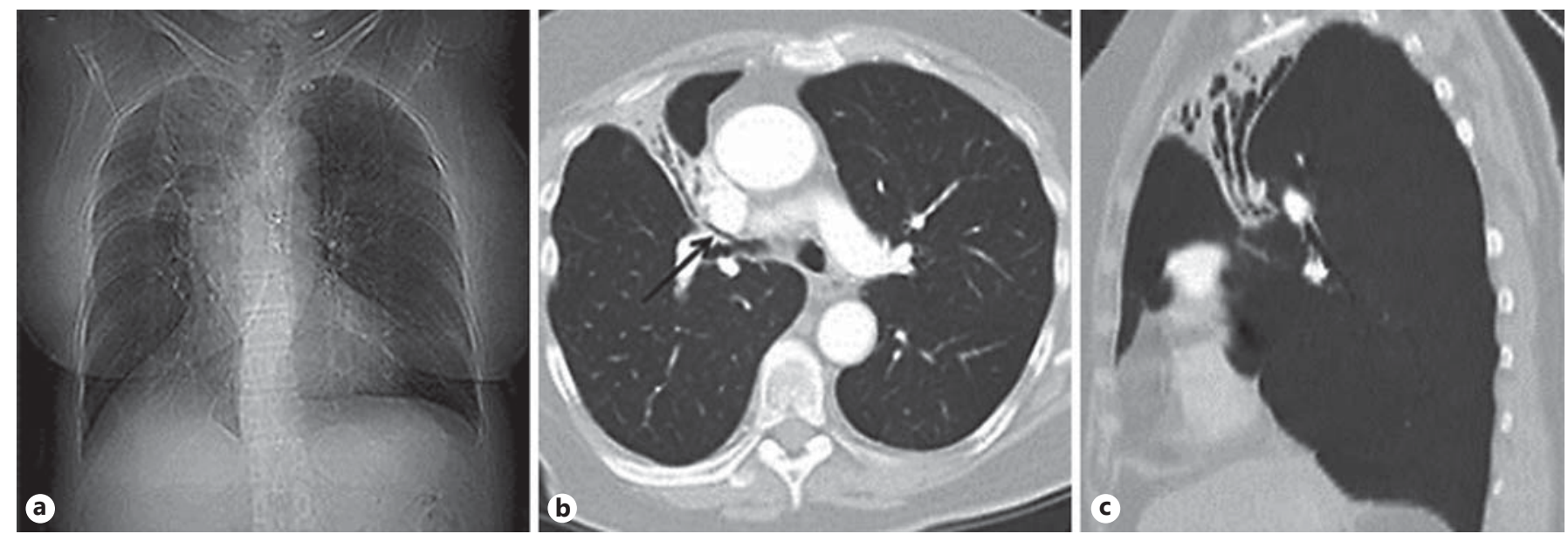

Fig. 1. a The CT scan showed bronchiectatic consolidation with air bronchogram formation involving the anterior segment of the upper lobe of the right lung with volume loss. Right deviation of the trachea was also demonstrated. Axial (b) and sagittal (c) CT scans showing bronchiectasis in the right upper lobe. The axial CT image also revealed compression of the bronchus of the right upper lobe (arrow).
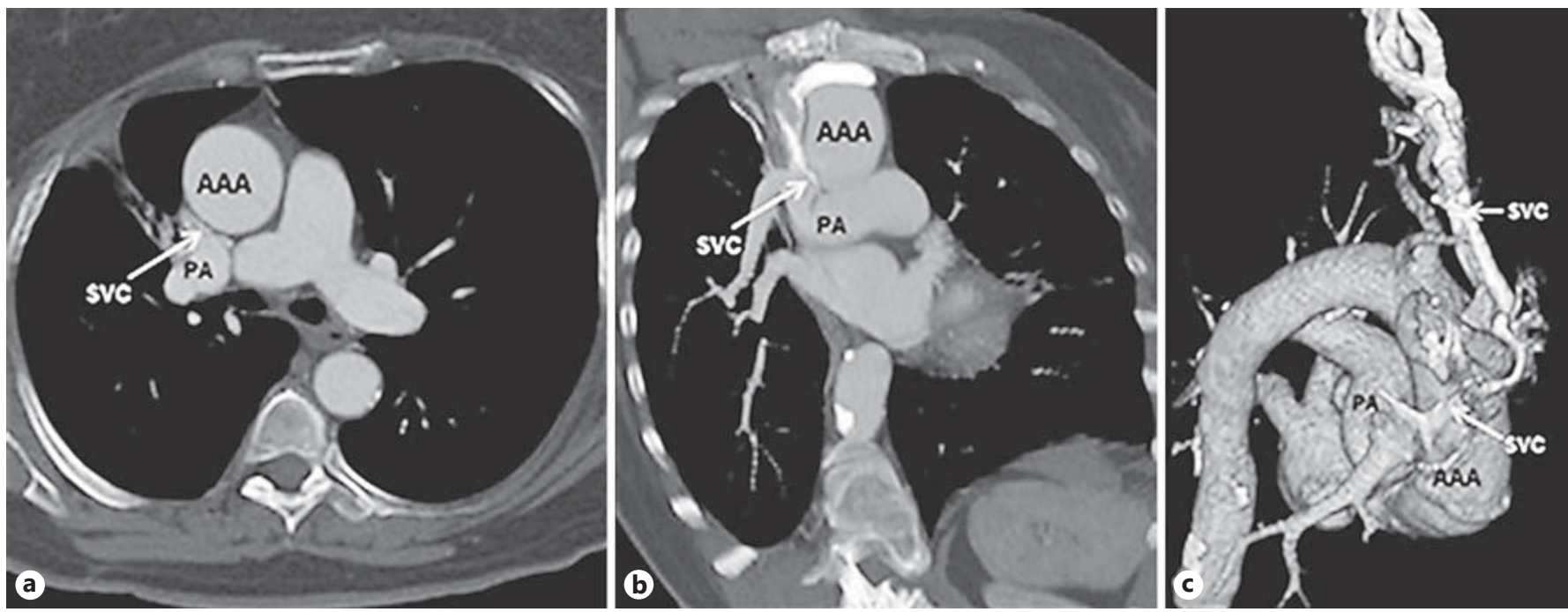

Fig. 2. Axial (a) and coronal oblique (b) CT scans revealed a compression of the superior vena cava (SVC) between an ascending aortic aneurysm (AAA) and the dilated right pulmonary artery (PA). c Volume-rendered 3D CT angiography showed an aneurys-

beats/min, her respiratory rate was 32 breaths/min and her temperature was $37.7^{\circ} \mathrm{C}$. During the pulmonary examination, a visual inspection of the chest revealed intercostal indrawing. Auscultation revealed rales at the right apex. Her right hemithorax was dull to percussion and respiratory rates were diminished. The rest of the examination was unremarkable.

A coronal computed tomography (CT; fig. 1a) showed bronchiectatic consolidation with air bronchogram formation involving the anterior segment of the upper lobe of the right lung with a $70 \%$ loss of volume. The right deviation of the trachea was also matic ascending aorta (AAA), right pulmonary artery kinking, poststenotic dilatation of the right pulmonary artery (PA), and compression of the superior vena cava. 3D CT angiography also revealed a dilatation of the proximal superior vena cava (SVC). demonstrated on the CT scan. Axial, sagittal and coronal oblique CT scans showed bronchiectasis in the right upper lobe (fig. 1b, c) and a poststenotic dilatation of the right pulmonary artery with a diameter of $32 \mathrm{~mm}$ (fig. 2a). The CT scan also revealed compression of the superior vena cava between an ascending aortic aneurysm and the dilated right pulmonary artery (fig. $2 a, b)$. There was compression of the bronchus of the right upper lobe in the CT image. A volume-rendered three-dimensional (3D) CT angiography image showed an aneurysmal ascending aorta, right pulmonary artery kinking, poststenotic dilatation of the right pulmonary ar- 
tery, and compression of the superior vena cava (fig. $2 \mathrm{c}$ ). The $3 \mathrm{D}$ $\mathrm{CT}$ angiography also revealed a dilatation of the proximal superior vena cava. A conventional right upper lobectomy was recommended to the patient, but she refused surgical therapy. Therefore, she was treated medically and monitored.

\section{Discussion}

This was a case of a rare coincidence of segmental PAE and superior vena cava compression. A CT scan revealed compression of the superior vena cava between an ascending aortic aneurysm and dilated right pulmonary artery, and also showed bronchiectasis secondary to compression of the bronchus of the right upper lobe, causing a segmental right PAE. Bronchiectasis due to pulmonary artery aneurysm has been reported previously in the English literature [4].

Causes of superior vena cava obstruction are thrombosis, fibrosis, external compression or neoplastic invasion [5]. These can be benign, malign or iatrogenic $[5,6]$. Vascular compression is a benign cause of superior vena cava obstruction. Ascending aortic aneurysms are often identified incidentally on chest radiography. A symptomatic presentation may often be due to the mass effect on neighboring anatomic structures such as the trachea and esophagus $[6,7]$, but, unlike our case, patients are rarely referred to the hospital with superior vena cava compression. Aortic aneurysms less than $5 \mathrm{~cm}$ in diameter can usually be treated conservatively and monitored [6].

Our case is unique in that the poststenotic dilated right pulmonary artery played a significant role in compressing the right upper lobe of the bronchus. Although aneurysm of the ascending aorta is a common subtype of thoracic aortic aneurysms, compression of the superior vena cava between the ascending aortic aneurysm and dilated pulmonary artery is an extremely rare condition [7].

In our case, the etiology was unknown. The etiology of PAE may relate to Marfan syndrome, valvular stenosis and infectious vasculitis, or it may be idiopathic [1-3]. A pulmonary artery aneurysm may present with symptoms such as dyspnea, chest pain, cyanosis, hemoptysis and cough [4]. In our patient, the poststenotic dilatation and ectasia of the right pulmonary artery caused increasing dyspnea and recurrent pneumonias due to stenosis of the right upper lobe of the bronchus. This case also presented the challenging management problem of a bronchiectatic right lung.

Bronchiectasis is mostly treated medically, reserving surgery for when medical treatment is ineffective. The purpose of surgical treatment for bronchiectasis is basically to provide a cure and to improve the quality of life after the failure of nonsurgical treatment. Moreover, surgical treatment aims to prevent severe pulmonary complications such as empyema, hemoptysis and abscess [4].

\section{Conclusion}

This case showed the importance of CT angiography in a case involving an unusual coincidence of segmental PAE and ascending aortic aneurysm. This coincidence was also associated with bronchiectasis and superior vena cava compression. CT angiography is the best investigational modality to confirm their existence and should be performed in patients with an especially recurrent pulmonary infection associated with a vascular anomaly.

\section{References}

1 Rose C, Wessel A: Three-decade follow-up in pulmonary artery ectasia: risk assessment strategy. Ann Thorac Surg 2002;73:973-975.

2 Bartter T, Irwin RS, Nash G: Aneurysms of the pulmonary arteries. Chest 1988;94:10651075.

3 Ugolini P, Mousseaux E, Sadou Y, et al: Idiopathic dilatation of the pulmonary artery: report of four cases. Magn Reson Imaging 1999; 17:933-937.

4 Arango TE, Cerezo MF, Salvatierra VA: Bronchiectasis due to pulmonary artery aneurysm.
Interact Cardiovasc Thorac Surg 2013;17: 176-178.

5 Turkyilmaz A, Aydin Y, Ogul H, et al: Total occlusion of the superior vena cava due to bronchogenic cyst. Acta Chir Belg 2009;109: 635-638.

6 Chao CT: Concurrent Salmonella mycotic abdominal aneurysm and empyema thoracis: a rare coincidence. Med Princ Pract 2014;23: 482-484.

7 Isselbacher EM: Thoracic and abdominal aortic aneurysms. Circulation 2005;111:816-828. 